\title{
Estrutura das exportações e crescimento econômico: uma análise empírica, $1985-2003^{1}$
}

\author{
Eva Yamila da Silva Catela ${ }^{2}$ \\ Gabriel Porcile ${ }^{3}$
}

\section{Resumo}

$\mathrm{O}$ artigo discute empiricamente a ideia de que a estrutura das exportações seja determinante para o crescimento econômico. A influência da estrutura das exportações sobre a taxa de crescimento é abordada no contexto de um modelo com restrição externa. Para testar essa relação, as exportações foram divididas em dois grupos: a) dinâmicas sob uma perspectiva schumpeteriana (que incluem setores de alta tecnologia) e b) dinâmicas sob uma perspectiva keynesiana (que incluem setores para os quais o crescimento da demanda internacional é superior à média). A hipótese-chave é que, quando os países nos quais os setores com eficiências keynesiana e schumpeteriana têm maior peso nas exportações, esses atingem taxas mais elevadas de crescimento. Os resultados mostram que os dois tipos de eficiência têm um efeito positivo e significativo, sendo mais forte o impacto da eficiência schumpeteriana.

Palavras-chave: Eficiência schumpeteriana; Eficiência keynesiana; Lei de Thirlwall; Crescimento econômico; Exportações.

\section{Abstract \\ Export structure and economic growth: an empirical analysis, 1985-2003}

The paper discusses the idea that the quality of exports matters for economic growth. The influence of the structure of exports on the rates of economic growth is discussed within the context of a growth model with external constraints. To test the relationship between exports and growth, exports are divided in two groups: a) dynamic from a Schumpeterian perspective (high-tech sectors) and b) dynamic from a Keynesian perspective (international demand grows at higher rates than the average). The key hypothesis is that countries whose total exports have higher shares of sectors with Keynesian and Schumpeterian efficiency will grow faster. The empirical results are consistent with this hypothesis. In addition, the results suggest that Schumpeterian efficiency tends to have a stronger impact on growth than Keynesian efficiency.

Key words: Schumpeterian efficiency; Keynesian efficiency; Thirlwall's law; Economic growth; Exports.

JEL F190, O140, C230.

(1) Trabalho recebido em novembro de 2008 e aprovado em maio de 2009.

(2) Professora Adjunta do Centro Sócio-Econômico da Universidade Federal de Santa Catarina (UFSC), Florianópolis, SC, Brasil. E-mail: evadasilvacatela@ gmail.com.

(3) Professor-Associado da Faculdade de Ciências Econômicas da Universidade Federal do Paraná (UFPR), Curitiba, PR, Brasil e pesquisador do CNPq (Conselho Nacional de Desenvolvimento Científico e Tecnológico). E-mail: porcile@ufpr.br. 
Eva Yamila da Silva Catela / Gabriel Porcile

\section{Introdução}

A relação entre exportações e crescimento econômico tem ocupado um espaço crescente na literatura econômica, sendo um tema central em diversos modelos heterodoxos de crescimento. Por um lado, os modelos de inspiração keynesiana enfatizam o papel da demanda externa como determinante último das taxas de crescimento (Mccombie; Thirlwall, 1994). A intensidade dessa demanda, por sua vez, depende da composição das exportações, que resulta numa maior ou menor elasticidade-renda das exportações. Por outro lado, modelos de inspiração schumpeteriana (Dosi et al., 1990; Araújo; Lima, 2007) têm enfatizado o papel das exportações de bens mais intensivos em tecnologia no crescimento. O papel desses setores é importante por duas razões: i) eles tendem a estar associados com elasticidades-renda da demanda mais elevadas; ii) eles geram externalidades e induzem processos mais intensos de aprendizado, que impulsionam os aumentos de produtividade e a competitividade internacional. ${ }^{4}$ Observe-se que os dois tipos de modelo são complementares e dialogam entre si. Os modelos schumpeterianos não negam o papel de fatores "puros de demanda" em certos episódios de crescimento (vinculados à commodity lottery, na expressão de Carlos DíazAlejandro), mas enfatizam seu vínculo com a dinâmica tecnológica no longo prazo.

Tendo como ponto de partida esse diálogo, o trabalho procura identificar os fatores que afetam o dinamismo exportador, combinando as visões keynesiana e schumpeteriana mencionadas acima. Com esse objetivo, as exportações foram divididas em dois tipos: de alta tecnologia, que estimulam taxas mais altas de aprendizado tecnológico (eficiência schumpeteriana); e as que mostram taxas de crescimento da demanda mundial acima da média (eficiência keynesiana). Por intermédio de técnicas de painel analisa-se em que medida a presença desses dois tipos de exportações afetou positivamente o crescimento econômico entre os anos 1985-2003, numa amostra de 107 países, dos quais 23 são industrializados e os restantes em desenvolvimento (incluindo países de América Latina, Ásia, Oceania, Europa e África). Assim, busca-se realizar uma contribuição à literatura empírica sobre crescimento com restrição externa, relacionando o crescimento às características da estrutura das exportações. ${ }^{5}$

O artigo está estruturado em duas seções além desta introdução e dos comentários finais. Na seção 1, é apresentado brevemente o modelo de Thirlwall, no qual o dinamismo das exportações é chave na determinação da taxa de crescimento de equilíbrio no longo prazo. Além disso, discute-se brevemente a

(4) Alguns trabalhos de inspiração neoclássica apontam nessa mesma direção; ver, por exemplo, Hausmann et al. (2005).

(5) A literatura sobre crescimento com restrição externa no Brasil já é ampla. Ver, por exemplo, Jayme (2003) e Carvalho (2008). 
relação existente entre o dinamismo tecnológico e o dinamismo exportador, assim como os conceitos de eficiência keynesiana e schumpeteriana do padrão de exportação. Na seção 2, apresentam-se brevemente diversos indicadores de capacidades tecnológicas e especialização. Existe uma ampla variação nesses indicadores entre os países da amostra, que acabam refletindo as assimetrias tecnológicas internacionais. Na sequência, realiza-se um teste empírico formal sobre a relação entre especialização e crescimento, usando técnicas de painel e de misturas finitas. A técnica de misturas finitas ainda não foi utilizada em testes de modelos de crescimento, podendo contribuir para uma estimação mais precisa dos parâmetros do modelo.

\section{Crescimento com restrição externa e padrão de especialização}

$\mathrm{Na}$ sua forma mais simples, o modelo de Thirlwall (1979) sugere que o crescimento da economia no longo prazo é definido pela razão entre a taxa de crescimento das exportações e a elasticidade-renda das importações. Essa seria a taxa compatível com o equilíbrio em conta corrente. Um país poderia crescer durante certo período a uma taxa superior à taxa de equilíbrio, mas a acumulação de déficits em conta corrente (e o consequente aumento da dívida externa) colocaria um freio nesse crescimento. ${ }^{6}$ Mais ainda, processos cumulativos poderiam dar lugar a círculos virtuosos ou viciosos, em que crescimento, investimento, progresso técnico e exportações se reforçam mutuamente (ver, por exemplo, Setterfield, 1997; Pugno, 1996; León-Ledesma e Thirlwall, 2002; um trabalho pioneiro nessa linha é Beckerman, 1962). Assim, por exemplo, num contexto de baixo crescimento das exportações, a capacidade subutilizada pode desencorajar o investimento e diminuir a incorporação de novas gerações de bens de capital, acentuando os problemas de competitividade.

O modelo de Thirlwall é derivado de equações de demanda de exportações e de importações de elasticidades constantes, mais a condição de equilíbrio em conta corrente. ${ }^{7} \mathrm{O}$ equilíbrio implica que:

(1) $P X=P * M E$

(6) Tal resultado pode ser visto como uma versão dinâmica do multiplicador de comércio exterior de Harrod (1933), segundo o qual o nível de renda de uma economia tende a ser idêntico ao nível das exportações dividido pela propensão marginal a importar. Também há uma forte relação desse modelo com a tradição estruturalista latino-americana (ver Rodriguez, 2007)

(7) A versão mais simples do modelo, que será usada neste trabalho, não considera os movimentos de capital, já que se supõe que entradas e saídas tendem a se compensar no longo prazo. Assim, o equilíbrio externo é visto apenas do ponto de vista da balança comercial. Alguns modelos têm estendido a equação (5) para incorporar nela a influência dos movimentos internacionais de capital e dos ciclos de endividamento externo (ver Moreno-Brid, 2003 e Barbosa-Filho, 2001). 
Eva Yamila da Silva Catela / Gabriel Porcile

$X$ e $M$ são quantidades exportadas e importadas, respectivamente, e respondem às seguintes funções de demanda:

(2) $M=\left(P^{*} E\right)^{\psi} P^{\phi} Y^{\pi}$

(3) $X=\left(\frac{P}{E}\right)^{\eta} P *^{\tau} Y * \varepsilon$

Na demanda por importações (2), $\psi$ é a elasticidade-preço das importações $(\psi<0) ; \phi$ é a elasticidade-preço cruzada; $\pi$ é a elasticidade-renda das importações $(\pi>0)$ e $Y$ é a renda interna. Na demanda por exportações (3), $\eta$ é a elasticidadepreço das exportações $(\eta<0)$; $\tau$ é a elasticidade-preço cruzada; $\varepsilon$ é a elasticidaderenda das exportações $(\varepsilon>0)$ e $Y^{*}$ é a renda externa.

Usando logaritmos e diferenciando com relação ao tempo, tem-se que:

(1a) $p+x=p^{*}+m+e$

(2a) $m=\psi p *+\psi e+\phi p+\pi y$

(3a) $x=\eta p-\eta e+\tau p^{*}+\varepsilon y^{*}$

As letras minúsculas nas equações anteriores representam taxas de crescimento. Substituindo as equações (2a) e (3a) na (1a) obtém-se a expressão que determina a taxa de crescimento do produto consistente com o equilíbrio no balanço de pagamentos:

(4) $y=\frac{p(1+\eta-\phi)-p^{*}(1-\tau+\psi)-e(1+\eta+\psi)+\varepsilon y^{*}}{\pi}$

Adicionalmente, são feitas duas hipóteses simplificadoras:

a) A elasticidade-preço da demanda por importações e exportações é igual a sua elasticidade-preço cruzada, isto é:

$\psi=\phi$

$\eta=\tau$

b) É válido o princípio da paridade do poder de compra na sua versão dinâmica; assim, no longo prazo, tem-se que:

$$
p^{*}+e-p=0
$$

A partir dessas hipóteses, a equação (4) pode ser simplificada, tendo como resultado:

(5) $y=\frac{\varepsilon y^{*}}{\pi}=\frac{x}{\pi}$ 
(5') $\frac{y}{y^{*}}=\frac{\varepsilon}{\pi}$

A expressão (5) exprime a relação entre crescimento do país, crescimento da economia mundial e as elasticidades-renda das exportações e importações. A expressão (5') exprime essa mesma relação em termos da taxa relativa de crescimento entre o país e o resto do mundo. Vale a pena lembrar que a equação (5') representa um fato estilizado ou regularidade empírica amplamente aceita na literatura. Mas como esse fato estilizado é interpretado do ponto de vista teórico, este é tratado como um motivo de controvérsia. Entre os autores de persuasão keynesiana, entende-se que a causalidade vai das elasticidades para o crescimento (Thirlwall, 2002; Mccombie; Thirlwall, 1994, capítulo 3). Inversamente, com base em um modelo ricardiano, Krugman (1988) sugere que as elasticidades-renda respondem passivamente ao crescimento da produtividade total dos fatores, sendo essa última variável o verdadeiro determinante do crescimento econômico.

Neste trabalho, assume-se que a determinação vai das elasticidades para o crescimento, como no modelo de Thirlwall, mas procura-se identificar os fatores que afetam tais elasticidades, em particular o papel que desempenha a tecnologia. Em outras palavras, admite-se que a tecnologia é um determinante central do crescimento econômico, mas que sua influência se exprime pelos seus efeitos sobre o padrão de especialização e sobre o dinamismo da demanda de exportações e importações. Nesse sentido, o modelo é keynesiano ou demand-led, na medida em que a influência da tecnologia sobre o crescimento é necessariamente mediada pela sua influência sobre a demanda. Alguns dos trabalhos que desenvolvem teoricamente essa ideia são Cimoli e Porcile (2009) e Araújo e Lima (2007). No terreno empírico, nosso approach desenvolve aquele recentemente sugerido por Cimoli et al. (2008).

Dosi et al. (1990) sugerem captar as inter-relações entre crescimento, tecnologia e demanda agregada por intermédio dos regimes que articulam esses diversos fatores (ver Diagrama 1). Três tipos de regimes podem ser identificados (Dosi et al., 1990):

- O regime tecnológico adotado por cada país, que depende da natureza das trajetórias tecnológicas, e da direção e intensidade dos feedbacks entre crescimento e mudança técnica.

- O regime de inserção na economia mundial, que exprime os efeitos da inovação sobre a competitividade, assim como a relação entre os setores tradable e non-tradable da economia. 
Eva Yamila da Silva Catela / Gabriel Porcile

- O regime de formação da demanda macroeconômica, que vincula a inovação às decisões de investimento e à operação do multiplicador e do acelerador keynesiano.

Diagrama 1

Relação entre inovação, competitividade e crescimento econômico

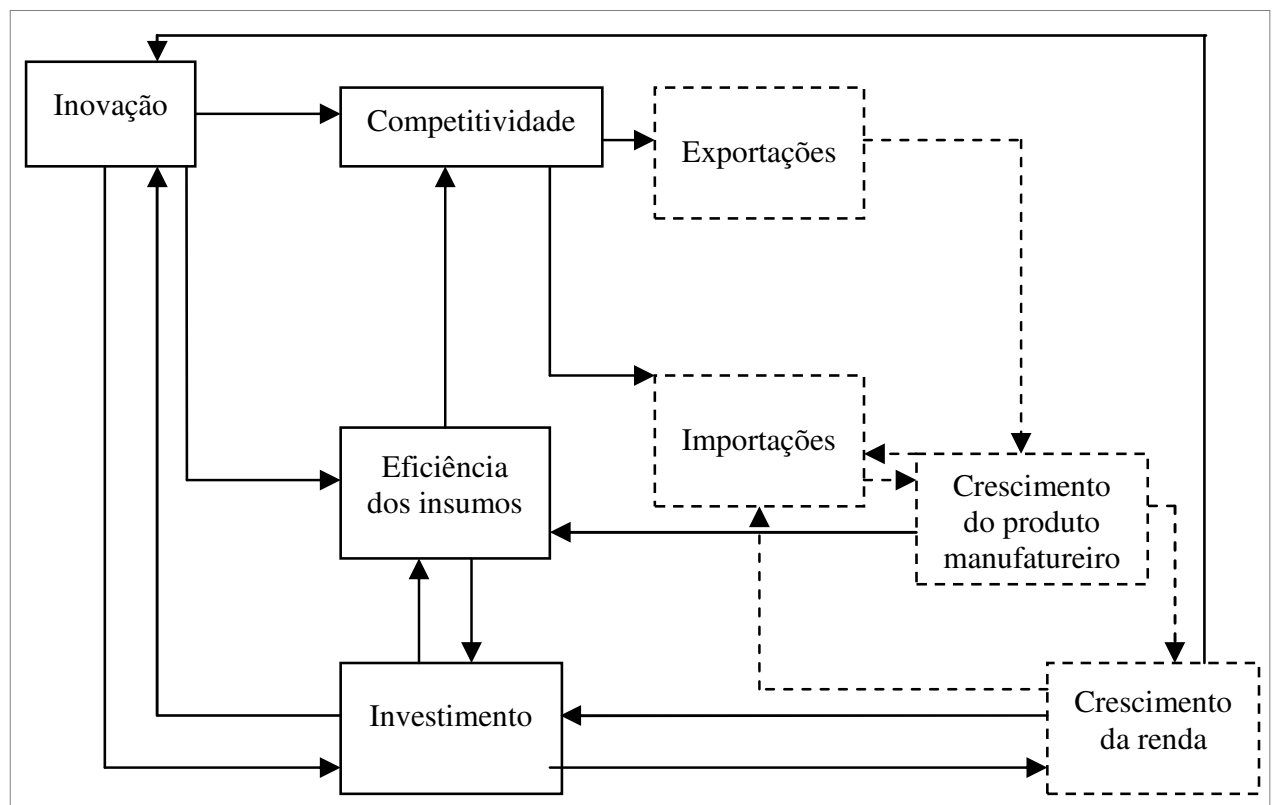

Fonte: Dosi et al. (1990).

Sem dúvida, esses distintos regimes estão fortemente inter-relacionados, mas neste trabalho interessa realizar um recorte específico, focando na relação entre o regime de inserção externa e os regimes tecnológicos e de demanda. Dosi et al. (1990) estabelecem uma tipologia de especialização internacional definida a partir dos padrões de alocação dos recursos produtivos. É geralmente aceito que sob as condições neoclássicas de rendimentos decrescentes e ausência de externalidades, o comércio internacional e seus resultados - a especialização de acordo com o princípio das vantagens comparativas - são eficientes. Dosi et al. (1990) chamam esse resultado de eficiência alocativa ou ricardiana. Todavia, esse tipo de eficiência deixa ainda duas questões em aberto. A primeira se relaciona com os efeitos que os padrões de alocação teriam sobre o dinamismo tecnológico; a segunda, com seus efeitos sobre o crescimento da demanda externa no longo prazo.

Seguindo esses autores, chamaremos o critério relacionado com o dinamismo inovador como "eficiência schumpeteriana", e o critério relacionado com a taxa de crescimento da demanda internacional como "eficiência 
keynesiana". O ponto-chave é que o mecanismo que garante a "eficiência ricardiana" não garante necessariamente que sejam alcançados os outros critérios de eficiência. Como discutido por Kaldor (1970) e Dosi et al. (1990), se diferentes setores apresentam diferenças significativas no seu "potencial dinâmico" (em termos de economias de escala, progresso técnico, possibilidades de divisão de trabalho, learning by doing, learning by using etc.), especializações que são eficientes no curto prazo podem (em certos casos) configurar círculos viciosos de retrocesso tecnológico relativo. ${ }^{8}$ Com efeito, o mecanismo de especialização internacional tem consequências dinâmicas, no sentido de que a especialização seleciona as áreas onde habilidades e conhecimentos serão acumulados (Archibugi; Michie, 1995; Miozzo; Walsh, 2006). Isso pode gerar trajetórias tecnológicas divergentes, com distinto potencial de crescimento e de inovação. As escolhas alocativas presentes influenciam a direção e as taxas futuras de mudança dos coeficientes técnicos (Arthur, 1994).

Um segundo aspecto tem a ver com o comportamento da demanda agregada. Ao abandonarmos o suposto de que os níveis de atividade econômica sempre correspondem aos de pleno-emprego no longo prazo (ou flutuam em torno desse nível) e caso seja admitido que as respostas aos desequilíbrios no comércio internacional ocorrem via produto (e não apenas via preços), então é necessário levar em conta o efeito da especialização sobre a demanda agregada (LeónLedesma; Thirlwall, 2002; Ocampo, 2005; Cimoli; Porcile, 2009). Quando as elasticidades-preço são demasiadamente baixas para compensar possíveis assimetrias nas elasticidades-renda, podem surgir problemas persistentes de desemprego.

Mercados descentralizados executam duas funções fundamentais. Primeiro, proveem mecanismos para coordenar decisões econômicas individuais e, fazendo isso, realocam recursos em direções que (sob as condições especificadas por cada teoria) apresentam distintos graus de eficiência. Segundo, proveem incentivos à inovação através da possibilidade da apropriação privada de seus benefícios, gerando diversas trajetórias tecnológicas, com suas características específicas de assimetria de informação, incerteza e irreversibilidade. Todavia, as características da tecnologia implicam a presença de distintos tipos de falhas de mercado, particularmente problemas de coordenação entre agentes heterogêneos. Por essa razão, além dos mercados, existem outras instituições que têm influência decisiva sobre a intensidade do aprendizado. O conceito de Sistema Nacional de Inovação (SNI) procura oferecer instrumentos analíticos para discutir essas

(8) Como se sabe, esse tema é extremamente importante nas teorias estruturalistas e nas teorias do desenvolvimento em geral. Ver, por exemplo, Ros (2000) e Prebisch (1981). 
instituições e como elas afetam a inovação e a difusão de tecnologia. ${ }^{9}$ Os países em desenvolvimento mostram SNIs imaturos (Fagerberg; Verspagen, 2007), que muitas vezes são incapazes de reverter processos cumulativos de aumento do hiato tecnológico com relação às economias industriais. A persistência no tempo das assimetrias tecnológicas entre os países é um indicador da dificuldade em alcançar os critérios schumpeterianos de eficiência, com as consequências negativas já mencionadas sobre o crescimento.

Em resumo, é necessário analisar se a eficiência ricardiana é compatível com as eficiências keynesiana e schumpeteriana, e se existem trade-offs entre elas no curto e no longo prazo. Na próxima seção, avalia-se empiricamente de que forma os distintos tipos de eficiência do padrão de especialização se vinculam com as variações nas taxas de crescimento econômico dos distintos países na economia internacional.

\section{Tecnologia e especialização: uma perspectiva comparada}

\subsection{Assimetrias tecnológicas e especialização}

A capacidade de inovação difere marcadamente entre países e isso guarda uma relação estreita com os padrões de especialização. Nesta seção apresentam-se dois indicadores de capacidades tecnológicas para 38 países do mundo, dos quais 20 são industrializados e 18 em desenvolvimento (ver Tabela 1), a saber, o World Economic Forum Technology Index (WEF) e o United Nations Development Program Technology Achievement Index (UNDP). O indicador WEF inclui 75 países e o UNDP inclui 72. Os anos incluídos na análise são 1997-2000 para o primeiro e 1995-2000 no segundo (ver Tabela 1).

Os indicadores são construídos combinando variáveis relativas à geração de tecnologia e inovação (patentes, artigos científicos, royalties), infra-estrutura e difusão de tecnologia (internet, PCs, consumo de energia), capital humano (anos de escolaridade, cursos de ciências) e competitividade. Os países são classificados em ordem decrescente segundo a posição de cada um deles dentro da classificação geral. Adicionalmente, na Tabela 1 encontram-se as exportações com eficiência schumpeteriana (exportações de alta tecnologia como percentual do total exportado) e as exportações com eficiência keynesiana (exportações dinâmicas como percentual do total exportado, em que as exportações dinâmicas são aquelas cuja demanda aumentou mais do que a média do comércio mundial). Os valores correspondem às médias do período 1985-2003. A tabela também apresenta as taxas de crescimento médio do PIB no período considerado.

(9) Sobre o conceito de Sistema Nacional de Inovação e como ele afeta o catching up tecnológico, ver Freeman (1988). 
Finalmente, na última coluna é apresentado o padrão de especialização predominante em cada país (média 1985-2003) a partir da composição das exportações, segundo a classificação do TradeCAN. Os grupos de produtos se definem do seguinte modo: Recursos Naturais (RRNN), que contém 45 produtos básicos (incluindo concentrados); manufaturas intensivas em recursos naturais (MRRNN), que inclui 35 produtos agropecuários/florestais e 30 associados a metais, petróleo e seus subprodutos, cimento e vidro; manufaturas de baixa tecnologia (MBT), que contém 20 produtos do encadeamento produtivo têxtil e 24 produtos de papel, vidro, aço e joias; manufaturas de média tecnologia, que inclui 5 produtos da indústria automotriz, 22 da indústria de processamento e 31 produtos da indústria de engenharia; e, por último, manufaturas de alta tecnologia (MAT), que contém 11 produtos da eletrônica, 7 produtos farmacêuticos, turbinas, aviões e instrumentos científicos.

Um primeiro aspecto a ressaltar é a alta concentração das capacidades inovadoras. ${ }^{10} \mathrm{O}$ grupo de países com maiores capacidades tecnológicas é bastante pequeno e se mantém ao longo do tempo, com poucos novos países ingressando nesse "clube" (o último a ingressar foi a Coreia), e poucas mudanças no ranking ao longo do tempo (Castaldi et al., 2004). Nos países industrializados, a especialização predominante é em manufaturas de média tecnologia e naquelas baseadas em recursos naturais. No caso dos países asiáticos, predomina a especialização em manufaturas de alta e baixa tecnologia. Na América Latina, a maioria dos países tem uma especialização baseada em recursos naturais, que em muitos casos também são suas exportações dinâmicas. ${ }^{11}$

Um segundo aspecto a ressaltar é que no caso das exportações dinâmicas no sentido keynesiano (EK) não se observam grandes diferenças na média por grupo de países. Sua participação é um pouco maior no caso da Ásia do que nos países industrializados e na América Latina. No entanto, quando os países são analisados individualmente, surgem diferenças marcantes. Em outras palavras, essas diferenças são maiores ao interior dos grupos do que entre os grupos. A

(10) Certamente, existem dificuldades muito grandes na mensuração da inovação tecnológica. Alguns autores optam por apresentar como proxy as patentes registradas nos Estados Unidos. Essa medida, no entanto, não considera importantes processos de aprendizado (muitas vezes informais) que afetam fortemente a competitividade e as exportações, mas que não geram patentes. Por essa razão, se usaram indicadores como o WEF e UNDP, que combinam um conjunto de variáveis, o que permitem reduzir as distorções dos indicadores individuais.

(11) Por exemplo: na Nicarágua, as exportações mais dinâmicas são duas: crustáceos e moluscos, e oleaginosas; no Peru, quatro das mais dinâmicas são recursos naturais: ouro, prata, crustáceos e moluscos e oleaginosas; no Equador, todas são recursos naturais: peixes, crustáceos e moluscos, e óleos de petróleo. O Chile mostra o mesmo padrão, no qual as exportações mais dinâmicas são recursos naturais: minerais de metais comuns, madeiras, peixes e polpa e desperdícios de papel, assim como Argentina (óleos de petróleo, óleo de origem vegetal, crustáceos e moluscos, milho), Brasil (oleaginosas, produtos derivados do petróleo, couros, carnes, polpa e desperdícios do papel) e Bolívia (gás natural, alimentos para animais e frutas e nozes). 
Eva Yamila da Silva Catela / Gabriel Porcile

análise da composição das exportações mostra variações importantes nas rubricas que respondem pelas exportações EK. Nos países latino-americanos, elas consistem de recursos naturais e suas manufaturas, enquanto no caso dos países asiáticos as mais dinâmicas são as manufaturas de alta e média tecnologia (com exceção de Sri Lanka, onde as exportações EK são de média e baixa tecnologia MBT - e de Indonésia, onde se destacam minerais de metais comuns, óleos de origem vegetal e MBT).

Tabela 1

Capacidade tecnológica, exportações de alta e média tecnologia e crescimento do PIB

\begin{tabular}{|c|c|c|c|c|c|c|}
\hline País & $\begin{array}{l}\text { Posição } \\
\text { WEF }\end{array}$ & $\begin{array}{l}\text { Posição } \\
\text { UNDP }\end{array}$ & $\begin{array}{c}\text { Exp. AT (\% } \\
\text { do total } \\
\text { exportado) }\end{array}$ & $\begin{array}{c}\text { Exp. DI } \\
(\% \\
\text { do total } \\
\text { exportado })\end{array}$ & $\begin{array}{l}\text { Crescimento } \\
\text { PIB (\%) }\end{array}$ & $\begin{array}{l}\text { Especialização } \\
\text { Predominante }\end{array}$ \\
\hline \multicolumn{7}{|l|}{ Industrializados } \\
\hline Estados Unidos & 1 & 2 & 28,73 & 33,52 & 2,99 & MAT \\
\hline Finlândia & 3 & 1 & 16,40 & 18,05 & 1,91 & MRRNN \\
\hline Suécia & 5 & 3 & 17,60 & 26,57 & 1,90 & MRRNN-MMT \\
\hline Canadá & 2 & 9 & 9,18 & 35,11 & 2,82 & MRRNN \\
\hline Austrália & 4 & 10 & 4,43 & 10,57 & 3,54 & RRNN \\
\hline Noruega & 6 & 12 & 4,38 & 51,17 & 3,22 & RRNN \\
\hline Japão & 19 & 4 & 29,95 & 26,10 & 1,34 & MMT \\
\hline Reino Unido & 8 & 7 & 22,61 & 28,87 & 2,31 & MRRNN-MAT \\
\hline Holanda & 11 & 6 & 16,01 & 14,07 & 2,28 & MRRNN \\
\hline Alemanha & 12 & 11 & 14,75 & 28,88 & 1,56 & MMT \\
\hline Israel & 21 & 18 & 19,35 & 48,79 & 4,21 & MRRNN \\
\hline Bélgica & 10 & 14 & 8,54 & 21,00 & 1,86 & MRRNN \\
\hline Nova Zelândia & 9 & 15 & 2,43 & 21,94 & 3,07 & RRNN \\
\hline Áustria & 13 & 16 & 11,90 & 17,92 & 2,05 & MMT \\
\hline França & 14 & 17 & 18,46 & 23,74 & 1,73 & MMT \\
\hline Irlanda & 23 & 13 & 31,57 & 45,17 & 1,56 & MAT \\
\hline Espanha & 22 & 19 & 8,54 & 12,19 & 2,62 & MMT \\
\hline Itália & 26 & 26 & 9,56 & 17,84 & 1,41 & MBT \\
\hline Portugal & 20 & 26 & 5,95 & 18,72 & 2,22 & MBT \\
\hline Grécia & 30 & 25 & 3,82 & 7,64 & 2,75 & MRRNN \\
\hline Média & & & 14,21 & 25,39 & 2,37 & \\
\hline \multicolumn{7}{|l|}{ Ásia } \\
\hline Coreia & 7 & 5 & 11,28 & 40,00 & 5,83 & MAT \\
\hline Singapura & 15 & 8 & 50,27 & 46,30 & 6,06 & MAT \\
\hline Malásia & 18 & 29 & 39,23 & 39,39 & 6,31 & MAT \\
\hline Tailândia & 31 & 36 & 24,73 & 22,52 & 4,67 & MAT \\
\hline Filipinas & 32 & 38 & 43,47 & 43,46 & 3,28 & MAT \\
\hline
\end{tabular}


Estrutura das exportações e crescimento econômico: uma análise empírica, 1985-2003

Tabela 1 - Continuação

\begin{tabular}{l|c|c|c|c|c|c}
\multicolumn{1}{c|}{ Tabela 1 - Continuação } & $\begin{array}{c}\text { Posição } \\
\text { WEF }\end{array}$ & $\begin{array}{c}\text { Posição } \\
\text { UNDP }\end{array}$ & $\begin{array}{c}\text { Exp. AT }(\% \\
\text { do total } \\
\text { exportado })\end{array}$ & $\begin{array}{c}\text { Exp. DI } \\
(\% \\
\text { do total } \\
\text { exportado })\end{array}$ & $\begin{array}{c}\text { Crescimento } \\
\text { PIB }(\%)\end{array}$ & $\begin{array}{c}\text { Especialização } \\
\text { Predominante }\end{array}$ \\
\hline China & 39 & 39 & 14,45 & 8,13 & 9,72 & MBT \\
\hline Índia & 44 & 46 & 3,30 & 12,44 & 5,61 & MBT \\
\hline Sri Lanka & 40 & 45 & 2,50 & 38,88 & 4,66 & MBT \\
\hline Indonésia & 41 & 44 & 4,47 & 12,52 & 4,27 & RRNN \\
\hline Média & & & 21,52 & 29,29 & 5,60 & \\
\hline
\end{tabular}

América Latina

\begin{tabular}{l|l|l|r|r|r|c}
\hline Argentina & 36 & 31 & 2,33 & 34,49 & 3,10 & RRNN \\
\hline Chile & 33 & 34 & 0,55 & 24,21 & 5,68 & MRRNN \\
\hline Costa Rica & 27 & 33 & 13,74 & 2,95 & 4,83 & MMT \\
\hline México & 29 & 30 & 19,03 & 30,20 & 2,85 & MMT \\
\hline Brasil & 37 & 37 & 5,35 & 19,70 & 2,31 & RRNN \\
\hline Peru & 42 & 41 & 0,55 & 14,67 & 3,78 & RRNN \\
\hline Bolívia & 45 & 40 & 0,39 & 40,33 & 3,41 & RRNN \\
\hline Equador & 46 & 42 & 0,63 & 38,99 & 2,28 & RRNN \\
\hline Nicarágua & 47 & 47 & 0,71 & 8,66 & 3,12 & RRNN-MBT \\
\hline Média & & & 4,80 & 23,80 & 3,48 & \\
\hline
\end{tabular}

Nota: "Exp. AT" são as exportações de alta tecnologia como percentual do total exportado na média entre 1985 e 2003. "Exp. DI" são exportações dinâmicas no sentido keynesiano (crescimento da demanda acima da média mundial), como percentual do total exportado na média entre 1985 e 2003. "WEF" e "UNDP" indicam a posição do país no ranking tecnológico de acordo com esses indicadores.

Fontes: Archibugi e Coco (2005), TradeCAN (2006), Penn World Table (2006).

Em resumo, em alguns países da Ásia e na América Latina, os setores de maior dinamismo de demanda são baseados em recursos naturais e suas manufaturas, assim como MBT. No caso de países industrializados, o dinamismo da demanda provém de produtos de classificações muito diversas, indicando uma estrutura exportadora altamente diversificada. $\mathrm{O}$ caso dos países africanos (não apresentados na Tabela 1, mas incluídos no teste empírico) é o mais preocupante. Eles caracterizam-se por uma diversificação exportadora quase nula e por estarem especializados em poucos recursos naturais, geralmente minerais, pedras e metais preciosos.

Com relação ao crescimento médio do PIB no período, observa-se que os países asiáticos alcançam as maiores taxas de crescimento. Os países industrializados mostram um crescimento menor, enquanto os latino-americanos apresentam um crescimento em níveis intermediários aos dos dois grupos 
anteriores. As exceções são Costa Rica e Chile, que apresentam um crescimento médio "asiático", mas com um padrão de especialização baseado em recursos naturais.

A seguir, procura-se relacionar o desempenho dos países em termos de crescimento econômico com sua estrutura exportadora, e em particular com as exportações de maior dinamismo tecnológico e de demanda - as eficiências schumpeteriana e keynesiana.

\subsection{Especialização e crescimento}

Como mencionado anteriormente, na sua versão mais simples a taxa de crescimento com equilíbrio no balanço de pagamentos é dada pela seguinte equação (ver seção 1):

$y=\frac{\varepsilon y^{*}}{\pi}=\frac{x}{\pi}$

Dado o objetivo deste trabalho (relacionar o desempenho competitivo dos países com a estrutura de suas exportações), interessa-nos decompor as exportações diferenciando as que são dinâmicas numa ótica schumpeteriana e keynesiana. $^{12}$ Relembrando as definições, as exportações com eficiência schumpeteriana são um indicador de dinamismo tecnológico, medido pela participação das exportações de alta tecnologia (em percentagem) no total de exportações. As exportações com eficiência keynesiana podem ser consideradas como um indicador de dinamismo da demanda, medido pela participação das exportações cuja demanda aumentou mais do que a média em relação ao comércio mundial nas exportações totais. Os dados dessas exportações têm como fonte o TradeCAN, versão 2006.

As equações estimadas foram as seguintes:

$$
\begin{aligned}
& y_{i}=\alpha_{i}+\beta\left(x_{S i}\right)+\pi\left(x_{K i}\right)+\psi\left(y^{*}\right)+e_{i} \\
& y_{i}=\alpha_{i}+\beta\left(x_{S i}\right)+\pi\left(x_{K i}\right)+\psi\left(y^{*}\right)+\lambda t_{o t}+e_{i}
\end{aligned}
$$

Onde $y$ e $y^{*}$ representam a taxa de crescimento real do produto per capita (do país i e da economia mundial, respectivamente) ${ }^{13}$ tot são os termos de troca, $x_{S}$ são as

(12) Um esforço nessa mesma direção foi realizado por Cimoli et al. (2008).

(13) Será utilizado como proxy do crescimento do PIB mundial o crescimento médio do PIB de 144 países do mundo (World Penn Table mark 6.0). 
exportações schumpeterianas, $x_{K}$ são as exportações keynesianas e $e$ é o termo de erro com as propriedades usuais. Os dados do PIB per capita real são do Penn World Table 6.2 (Heston et al., 2006). Os dados dos termos de intercâmbio de todos os países provêm do WDI. ${ }^{14}$

Ao discutir as implicações de seu modelo, Thirlwall (1979) adota a hipótese de que os termos de troca podem flutuar no curto prazo, mas que são aproximadamente constantes no longo prazo. Como foi oportunamente sugerido por Lopez e Cruz (2000) para o caso de América Latina, os termos de troca apresentaram importantes flutuações no período considerado. Essas flutuações podem ter afetado a competitividade dos bens tradables e, consequentemente, o produto em equilíbrio externo. Por essa razão, os termos de troca foram incluídos na estimação do modelo, de forma a evitar problemas de especificação. ${ }^{15}$

A estimação do modelo foi realizada através de três tipos de técnica: i) pooled OLS; ii) least square dummy variables, efeitos fixos (LSDV-FE); e iii) efeitos aleatórios (GLS-RE). A estimação mediante efeitos fixos específicos para cada país é importante e necessária para controlar as diferenças entre eles. Foi realizada também uma regressão pooled que remove os efeitos fixos dos países, como forma de comprovar que os resultados obtidos não representam um "artifício" da metodologia de efeitos fixos.

Com relação aos efeitos aleatórios, sua validade foi testada através do teste de Hausman. Esse teste tem como hipótese nula a ausência de diferenças sistemáticas entre os parâmetros de um modelo de efeitos fixos e de um modelo de efeitos aleatórios. A estatística do teste é dada por:

$$
\chi^{2}=\left(\hat{\beta}_{F E}-\hat{\beta}_{R E}\right) \cdot\left[V\left(\hat{\beta}_{F E}\right)-V\left(\hat{\beta}_{R E}\right)\right]^{-1}\left(\hat{\beta}_{F E}-\hat{\beta}_{R E}\right)
$$

Num primeiro momento, foi realizada a estimação para o total dos países e o total do período (1985-2003), como pode ser visto na Tabela 2. Os resultados reportados são consistentes com o previsto pelo modelo de Thirlwall ampliado para incluir a dimensão tecnológica da especialização.

(14) Os termos de troca representam a razão entre o nível de preços de importação e o nível de preços de exportação.

(15) O objetivo deste trabalho não é analisar os resultados associados aos termos de troca, mas a omissão desta variável poderia levar a uma incorreta especificação do modelo.

Economia e Sociedade, Campinas, v. 19, n. 2 (39), p. 291-313, ago. 2010. 
Eva Yamila da Silva Catela / Gabriel Porcile

Tabela 2

Estimações para o período 1985-2003 e todos os países

Todos os países: resultados das estimações

\begin{tabular}{|c|c|c|c|}
\hline & Pooled OLS & LSDV-FE & GLS-RE \\
\hline \multirow[t]{2}{*}{ ßo } & $8,9197 * * *$ & $8,2762 * * *$ & $8,2559 * * *$ \\
\hline & $(0,1487)$ & $(0,0258)$ & $(0,1080)$ \\
\hline \multirow[t]{2}{*}{ exdin } & $0,0045 * * *$ & $0,0012 * * *$ & $0,0012 * * *$ \\
\hline & $(0,0019)$ & $(0,0002)$ & $(0,0002)$ \\
\hline \multirow[t]{2}{*}{ exat } & $0,0329 * * *$ & $0,0142 * * *$ & $0,0144 * * *$ \\
\hline & $(0,0021)$ & $(0,0008)$ & $(0,0008)$ \\
\hline \multirow[t]{2}{*}{ var_pibm } & 0,0274 & $0,0104 * * *$ & $0,0104 * * *$ \\
\hline & $(0,0318)$ & $(0,0047)$ & $(0,0047)$ \\
\hline \multirow[t]{2}{*}{ tot } & $-0,0087 * * *$ & $-0,0003 * *$ & $-0,0003 * *$ \\
\hline & $(0,0009)$ & $(0,0001)$ & $(0,0001)$ \\
\hline $\mathrm{R}^{2}$ & 0,18 & 0,19 & 0,19 \\
\hline $\mathrm{F}$ & 92,17 & 92,24 & - \\
\hline Wald $\chi^{2}$ & - & - & 376,78 \\
\hline Observações & 1649 & 1649 & 1649 \\
\hline
\end{tabular}

Nota: Erro Padrão entre parênteses.

***Significativo ao nível de $1 \%$. **Significativo ao nível de $5 \%$.

Fonte: Elaboração dos autores, a partir dos dados do TradeCAN, WDI e Penn World Table, mark 6.

a) $\mathrm{O}$ crescimento do PIB per capita encontra-se positivamente relacionado com as exportações de alta tecnologia e com as exportações dinâmicas. As exportações de alta tecnologia apresentam um coeficiente mais elevado do que as exportações dinâmicas no sentido keynesiano. Os coeficientes das duas variáveis relativas ao padrão de especialização têm o sinal esperado e são significativos em nível de $1 \%$.

b) Já no caso da variação do PIB mundial, ela tem uma influência positiva sobre o crescimento, como esperado, sendo os resultados significativos em todos os casos, com exceção do caso de Pooled OLS. O impacto positivo de todas as variáveis diminui (coeficientes menores) quando são considerados os efeitos fixos e aleatórios, metodologias mais adequadas para esse caso.

c) O coeficiente da variável "termos de troca" apresenta o sinal esperado (negativo), o que estaria de acordo com a condição de Marshall-Lerner (a melhora nos termos de troca afeta negativamente o saldo da balança comercial). 
d) $\mathrm{O}$ grau de ajuste do modelo $\left(\mathrm{R}^{2}\right.$ de aproximadamente $20 \%$ para todas as estimações) pode ser considerado satisfatório, dado o nosso objetivo principal testar a existência de uma relação entre especialização e crescimento.

O teste para a escolha entre os modelos de efeitos fixos e efeitos aleatórios (teste de Hausman) indica que o primeiro modelo é o mais adequado. Com efeito, obtém-se uma estatística $\chi^{2}$ de 99,34, o que permite rejeitar a hipótese nula de que as diferenças entre os coeficientes não são sistemáticas (ver Apêndice 2). Pelo contrário, o teste $\mathrm{F}$ para ausência de efeitos fixos sugere que esses efeitos são significativos, com um p-valor significante em nível de $1 \%$.

A seguir, a estimação do modelo será realizada usando a metodologia de misturas finitas. A vantagem dessa metodologia é que permite estimar os parâmetros do modelo para grupos de países que mostram entre si maior grau de similaridade. Existindo diferenças estruturais entre esses grupos, a metodologia de misturas finitas permitiria controlá-las e realizar estimações com base em grupos mais homogêneos.

\subsection{Uma aplicação do modelo de misturas finitas}

Nesta seção considera-se o problema de estimar um modelo de regressão linear para dados em painel, sob a hipótese de que os dados são gerados a partir de uma função de densidade de misturas finitas, caracterizada por valores de parâmetros diferentes para cada componente ou grupo dentro da distribuição. A estimação a partir da hipótese de densidade de probabilidade simples pode resultar em parâmetros viesados: por essa razão, é preferível modelar a distribuição estatística a partir de uma mistura de outras distribuições, obtendo assim uma maior homogeneidade na amostra.

Pesaran e Smith (1995) apresentam quatro formas diferentes para modelar a possível heterogeneidade da distribuição: (a) estimar regressões separadas por grupos; (b) possibilitar interceptos fixos ou aleatórios, mas com uma inclinação igual por grupos; (c) fazer uma média dos dados por grupo e especificar um modelo agregado de séries temporais; e (d) fazer um média temporal e estimar regressões sobre as médias dos grupos. O trabalho de Kaplan (2005) difere dos anteriores na medida em que utiliza o modelo de misturas finitas para obter diferentes valores dos parâmetros da regressão (intercepto; inclinação; variâncias) para cada grupo dentro da distribuição.

Para entender melhor o significado da estimação através das misturas finitas, tomar-se-á como exemplo um modelo simples, a saber, um modelo linear de duas variáveis, $z_{i}=\left(y_{i} w_{i}\right)$, onde $y_{i}$ é a variável endógena e $w_{i}$ é a variável exógena. Formalmente: 
Eva Yamila da Silva Catela / Gabriel Porcile

$$
f\left(z_{i}\right)=f\left(y_{i} / w_{i}\right) f\left(w_{i}\right)
$$

onde $f\left(y_{i} / w_{i}\right)$ é a função de densidade condicional (regressão) e $f\left(w_{i}\right)$ é a distribuição marginal da variável exógena. $\mathrm{O}$ modelo de regressão para mistura finita pode ser escrito como:

$$
y_{i k}=\alpha_{k}+\Delta_{k} w_{i k}+u_{i k} \quad i=1,2, \ldots, \mathrm{n} ; \mathrm{k}=1,2, \ldots, \mathrm{K}
$$

onde $\alpha_{k}$ é o intercepto para o componente k, $y_{i k}$ é a variável endógena para o país i no componente $\mathrm{k}, w_{i k}$ é a variável exógena para o país i no componente $\mathrm{k}$, com peso $\Delta_{k}$ (que varia para cada componente), $u_{i k}$ é o termo de erro cuja variância $\sigma_{i k}^{2}$ é assumida normal e homocedástica dentro dos componentes, mas possivelmente heterocedástica entre componentes.

No contexto da discussão desenvolvida na seção anterior, a utilização do modelo de distribuição de misturas finitas normais ${ }^{16}$ no modelo de painel é um instrumental que permite, a partir dos dados das variáveis de especialização comercial (eficiências keynesiana e schumpeteriana), identificar diferentes intensidades de resposta do crescimento do PIB sem ter de separar arbitrariamente os grupos na forma tradicional (por exemplo, industrializados e em desenvolvimento). A estratégia de estimação implica, em primeiro lugar, fazer uma avaliação acerca do número de grupos que os dados suportam. Na Tabela 3 são apresentados dois critérios de qualidade de ajuste em modelos para um componente (que corresponde a uma estimação OLS convencional), e para dois e três componentes (que correspondem a estimações FMM, finite mixture model). Esses critérios são o Critério de Informação de Akaike (AIC, Akaike, 1973) e o Critério de Informação Bayesiano (BIC, Schwarz, 1978).

Tabela 3

Critérios de Informação de Akaike e Schwartz para modelos de distintos números de componentes

\begin{tabular}{l|c|c}
\hline \multicolumn{1}{c|}{ Modelo } & AIC & BIC \\
\hline OLS um componente & $4.863,73$ & $4.890,78$ \\
\hline FMM dois componentes & $4.552,11$ & $4.622,41$ \\
\hline FMM três componentes & $4.295,70$ & $4.403,86$ \\
\hline
\end{tabular}

Fonte: Elaboração dos autores, a partir dos dados do TradeCAN, WDI e Penn World Table, mark 6.

(16) O modelo permite a estimação da regressão supondo diversos tipos de distribuição (Normal ou Gaussiana, Poisson, Gama, Binomial negativa, t-Student, Weibull). Neste trabalho é utilizada a função normal para a mistura na distribuição. 
Deve ser lembrado que quanto menor o valor de AIC e BIC melhor o ajuste do modelo. Os resultados encontrados apontam que os dados se ajustam melhor ao modelo de misturas finitas de três componentes. Outra prova que reforça esse resultado é o teste LR. Se a função de densidade de probabilidade $\mathrm{f}_{\mathbf{y}}$ toma a forma de uma densidade de misturas para todo $\mathbf{y} \in y$ :

$$
f_{y}=\eta_{1} f_{1}(y)+\ldots+\eta_{K} f_{K}(y)
$$

onde $f_{K}(y)$ é a função de densidade de probabilidade para todo $\mathrm{k}=1, \ldots, \mathrm{K}$. A densidade $f_{K}(y)$ é chamada de densidade componente, sendo $\mathrm{K}$ o número de componentes. Os parâmetros $\eta_{1}, \ldots, \eta_{K}$ são os pesos, e o vetor $\eta=\left(\eta_{1}, \ldots, \eta_{K}\right)$ é chamado de distribuição de pesos.

No teste, as hipóteses nula e alternativa são, respectivamente, que $f_{Y}$ têm dois componentes e que $f_{Y}$ tem três componentes. $O$ resultado do teste indica um valor da estatística $\chi^{2}$ igual a 270,41 , sendo então rejeitada a hipótese nula de que existem apenas dois componentes dentro da nossa amostra.

A Tabela 4 apresenta os resultados da regressão de misturas finitas para três componentes amostrais. Um primeiro resultado importante é a configuração dos grupos. Cada grupo conta com $22 \%, 65 \%$ e $13 \%$ da população amostral, ou seja, há uma distribuição observacional trimodal com um maior número de países no segundo grupo. Todos os três grupos apresentam um coeficiente positivo e significativo de resposta do crescimento às exportações de alta tecnologia. Para o grupo 2, que representa $65 \%$ da população da amostra, o coeficiente é maior em relação aos outros grupos, sendo que o grupo 3, de menor população, apresenta o coeficiente menor. O coeficiente de resposta do crescimento para o grupo 2 é maior quando a estimação se faz por misturas finitas, sobretudo quando se realiza painel com efeitos fixos, mas no caso dos outros grupos o coeficiente é menor. As exportações dinâmicas no sentido keynesiano não são significativas para o grupo 1, mas são significativas e positivas para os outros dois grupos. $\mathrm{O}$ maior coeficiente é observado no grupo 3, que apresenta por sua vez o menor coeficiente de exportações de alta tecnologia. Esse resultado contrasta com o observado para o grupo 2, que tem o maior coeficiente nas exportações de alta tecnologia e o menor nas exportações keynesianas. Comparando com os resultados obtidos através dos efeitos fixos, é possível notar que para os grupos 2 e 3 o coeficiente é bastante maior no caso do modelo de misturas finitas.

Os termos de troca apresentam o sinal negativo esperado, já encontrado nas estimações de painel anteriores, e os maiores coeficientes são observados nos grupos 2 e 3 . O coeficiente é maior para todos os grupos considerados separadamente, quando comparados àqueles considerados juntos na estimação de 
efeitos fixos. A variação do PIB mundial apresenta coeficiente positivo para todos os grupos, mas só resulta significativo no caso do grupo 3.

A média da variável dependente (PIB per capita real) ${ }^{17}$ para cada grupo é apresentada na tabela e permite inferir alguns resultados importantes. A partir dessas médias, dos erros padrão estimados e das probabilidades de ocorrência de cada componente, podemos identificar a que grupo pertence cada observação. Os países da América Latina (México, Brasil, Argentina, Colômbia, Chile, Paraguai, Peru, Uruguai, Venezuela, Equador, Panamá, Costa Rica) permanecem no grupo intermediário ao longo de todo o período, sem mostrar uma mudança importante dentro do grupo, com exceção do Chile que passa da média do grupo para o limite superior (o PIB per capita médio do Chile aumenta de US\$ 5.700 em 1985 para US\$ 8.500 em 2003). Bolívia e Nicarágua caem dentro do grupo e se posicionam no limite inferior em 2003, enquanto a Bolívia se posiciona na seção de transição entre o grupo 2 e o grupo 1.

Alguns países asiáticos merecem destaque, dado que mudam de grupo ao longo do período observado. A China parte em 1985 no grupo 1, com uma renda per capita de US\$1.131, passando a partir dos anos 1998-1999 para o grupo intermediário com uma renda per capita de quase US\$ 5.000. Um caso similar é o da Indonésia, que começa no limite superior do primeiro grupo e se insere no grupo intermediário a partir de 1992. A Coreia transita do grupo intermediário para o grupo rico em 2000. A Malásia tinha em 1985 um PIB per capita similar à média do grupo intermediário e se encontra em 2003 muito próxima do grupo dos países ricos. ${ }^{18}$ A Tailândia encontrava-se no início no limite inferior do grupo e termina no limite superior. Os países estagnados no primeiro grupo são na sua maioria da África e da Ásia, como por exemplo, Mali, Benin, Bangladesh, Burkina Faso, República da África Central, Costa do Marfim, Moçambique, Níger e Nigéria, Paquistão, Uganda, entre outros. Já os países desenvolvidos encontram-se no grupo 3, sendo que alguns deles entram nesse grupo ao longo do período analisado, como Irlanda e Espanha (que começam em 1985 no limite superior do grupo intermediário e passam para o grupo "rico" entre 1995 e 1996). Portugal e Grécia apenas ingressam nesse grupo no final do período analisado.

(17) Na estimação foi utilizado o logaritmo natural do PIB per capita real, mas na tabela apresentam-se os valores "recuperados" para visualizar diretamente os PIB médios de cada grupo.

(18) Dado que se trata de resultados probabilísticos e que os intervalos não são mutuamente excludentes, certas observações não podem ser adjudicadas a nenhum grupo com total certeza. A observação da Malásia para o ano 2003 é um desses casos. 
Tabela 4

Estimação de regressão com misturas finitas para o período 1985-2003 e três grupos de países

\begin{tabular}{l|r|r|r}
\hline & Grupo 1 & Grupo 2 & \multicolumn{1}{c}{ Grupo 3 } \\
\hline Bo & $7,0786 * * *$ & $8.9139 * * *$ & $10,5078 * * *$ \\
\hline Exdin & $(0,1172)$ & $(0,1523)$ & $(0,09641)$ \\
\hline & $-0,0003$ & $0,0039 * *$ & $0,0074 * * *$ \\
\hline Exat & $(0,0014)$ & $(0,0019)$ & $(0,0008)$ \\
\hline & $0,0070 * * *$ & $0,0388 * * *$ & $0,0039 * * *$ \\
\hline var_pibm & $(0,0012)$ & $(0,0026)$ & $(0,0010)$ \\
\hline & 0,0039 & 0,0406 & $0,0203 * *$ \\
\hline Tot & $(0,0262)$ & $(0,0326)$ & $(0,0116)$ \\
\hline & $-0,0020 * * *$ & $-0,0075 * * *$ & $-0,0071 * * *$ \\
\hline Média da variável dependente & $(0,0007)$ & $(0,0009)$ & $(0,0009)$ \\
\hline Proporção da amostra & $1.021,85$ & $5.436,67$ & $20.699,13$ \\
\hline Wald $\chi^{2}$ & $21,67 \%$ & $64,97 \%$ & $13,36 \%$ \\
\hline AIC & & - & 490,5 \\
\hline BIC & & - & $4.548,09$ \\
\hline Obs. & & & $4.618,39$ \\
\hline Font Elaboç & & & 1.649 \\
\hline
\end{tabular}

Fonte: Elaboração dos autores, a partir dos dados do TradeCAN, WDI e Penn World Table, mark 6.

Obs.: Erro Padrão em parênteses.

***Significativo ao nível de $1 \%$. **Significativo ao nível de $5 \%$.

Em resumo, os resultados encontrados são em geral favoráveis à perspectiva que enfatiza o diálogo entre modelos keynesianos e schumpeterianos, onde tecnologia e dinamismo exportador se articulam para sustentar o crescimento econômico de longo prazo. Os coeficientes foram significativos e mostraram os sinais esperados com os distintos métodos de estimação. Assim, os resultados são compatíveis com a hipótese de que a especialização é um importante fator para o crescimento. $\mathrm{O}$ uso de misturas finitas possibilitou não apenas identificar distintos grupos de países segundo sua renda média, como também identificar mudanças de posição ao longo do tempo. No caso dos países latino-americanos, eles se localizaram num grupo intermediário e permaneceram ali, sem que se observem os processos de convergência na direção do grupo 1 que ocorreram na Ásia. 
Eva Yamila da Silva Catela / Gabriel Porcile

\section{Comentários finais}

O objetivo do trabalho foi avaliar a relação existente entre crescimento econômico e padrão de especialização, enfatizando o papel das exportações dinâmicas do ponto de vista da tecnologia e da expansão da demanda. As primeiras se relacionam com a eficiência schumpeteriana e as segundas com a eficiência keynesiana do padrão de especialização. Com efeito, por um lado, esse padrão afeta a inovação tecnológica, já que define as áreas onde se acumulam as capacidades tecnológicas. Surgem assim trajetórias tecnológicas que mostram distinto potencial de geração de inovações, externalidades e aumentos de produtividade. Por outro lado, alguns padrões de especialização beneficiam-se de taxas de crescimento da demanda mais intensos, capazes de induzir círculos virtuosos de expansão do investimento e do emprego. Ambos os fatores afetam dessa forma a taxa de crescimento de longo prazo da economia compatível com o equilíbrio externo.

A relação entre especialização e crescimento foi testada econometricamente, usando distintas metodologias: pooling, painel com efeitos fixos, painel com efeitos aleatórios e painel com misturas finitas. Essa última metodologia permite identificar diferenças nos parâmetros do modelo entre grupos definidos estatisticamente a partir dos próprios dados da amostra - e não a partir de algum critério exógeno de classificação. Todas as metodologias indicam que os dois tipos de indicadores da qualidade do padrão de especialização utilizados na análise - participação das exportações de alta tecnologia e participação das exportações com crescimento da demanda acima da média - tiveram uma influência positiva e estatisticamente significativa sobre a taxa de crescimento da economia. Também os termos de troca tiveram uma influência sobre o crescimento, com sinal negativo, conforme esperado quando se assume válida a condição de Marshall Lerner.

O painel de misturas finitas separou os países em três grupos e permitiu estimar os parâmetros do modelo com base em grupos mais homogêneos. Surgem desse processo algumas diferenças nos coeficientes, que não afetam, no entanto, as conclusões obtidas com a estimação de painel. Um resultado interessante é que apesar de os países asiáticos e América Latina se encontrarem na sua maioria no grupo intermediário em termos de renda per capita, os primeiros apresentam um comportamento dinâmico, chegando até o grupo superior ou avançando do limite inferior para o superior dentro do grupo. Oslatino-americanos, por sua vez, mostram certa estagnação, não mudando em geral (a exceção é Chile) sua posição dentro do grupo.

Os resultados são favoráveis para uma leitura da Lei de Thirlwall na qual as dimensões schumpeterianas e keynesianas se conjugam. Tanto a eficiência 
keynesiana como a schumpeteriana do padrão de especialização revelaram-se significativas, mas os efeitos positivos das capacidades tecnológicas foram mais fortes, em termos quantitativos, do que a sorte na commodity lottery. Países que são capazes de mostrar uma presença significativa de exportações de alta tecnologia conseguem responder e ajustar-se melhor às mudanças e desafios do comércio internacional. Mais ainda, há uma sobreposição elevada entre exportações dinâmicas e exportações de alta tecnologia, na medida em que as exportações de maior intensidade tecnológica tendem também a ser mais dinâmicas. Nesse sentido, a "velha" preocupação estruturalista com a composição das exportações, e particularmente com a intensidade tecnológica, confirma-se à luz de seu vínculo com o crescimento econômico.

\section{Bibliografia}

ARAÚJO, A.; LIMA, G. T. A structural economic dynamics approach to balance-ofpayments-constrained growth. Cambridge Journal of Economics, v. 31, p. 755-774, 2007.

AKAIKE, H. Information theory and an extension of the maximum likelihood principle. In: PETROV, B. N.; CSAKI, F. (Ed.). Second international symposium on information theory, 1973.

ARCHIBUGI, D.; COCO A. Measuring technological capabilities at the country level: a survey and a menu for choice. Research Policy, 34, 2005.

; MICHIE, J. The globalisation of technology: a new taxonomy. Cambridge Journal of Economics, v. 19, n. 1, p. 121-140, Feb. 1995.

ARTHUR, B. A. Increasing returns and path-dependency in economics. Michigan University Press, 1994.

BARBOSA-FILHO, N. The balance-of-payments constraint: from balanced trade to sustainable debt. Banca Nazionale del Lavoro Quarterly Review, n. 219, Dec. 2001.

BECKERMAN, W. Projecting Europe's growth. Economic Journal, n. 73, p. 912-925, 1962.

CAPDEVIELLE, M. Globalización, Especialización y Cambio Estructural en América Latina. In: CIMOLI, M. (Ed.). Heterogeneidad estructural, asimetrías tecnológicas y crecimiento en América Latina. Santiago: BID-CEPAL, 2005.

CARVALHO, V. A restrição externa e a perda de dinamismo da economia brasileira: investigando as relações entre estrutura produtiva e crescimento econômico. $1^{\circ}$ lugar Prêmio BNDES, 2008.

CASTALDI, C.; CIMOLI, M.; CORREA, N.; DOSI, G. Technological learning, policy regimes and growth in a 'globalized' economy: general patterns and the Latin American experience. LEM Working Paper Series 01, 2004.

CIMOLI, M.; PORCILE, G. Growth, wage bargaining and technological policy in a BOPconstrained multigoods growth model. Metroeconomica, Special issue, forthcoming, doi: 10.1111/j.1467-999X.2009.04066.x, 2009. 
Eva Yamila da Silva Catela / Gabriel Porcile

; PORCILE, M. e ROVIRA, S. Structural change and the balance-of-payments constraint: why did Latin America fail to converge? Cambridge Journal of Economics, p. 1-23, doi:10.1093/cje/ben060, 2008.

DOSI, G., PAVITT, K.; SOETE, L. The economics of technical change and international trade. New York: Harvester Wheatsheaf, 1990.

FAGERBERG, J. Technology and international differences in growth rate. Journal of Economic Literature, n. 32, p. 1147-1175, 1994.

; VERSPAGEN, B. Innovation, growth and economic development: Have the conditions for catch-up changed? International Journal of Technological Learning, Innovation and Development, v. 1, n. 1, 2007.

FREEMAN, C. Technical change and economic theory. London: Pinter Publishers, 1988.

HERNÁNDEZ, R. ¿Se erosiona la competitividad de Centroamérica y la República Dominicana con el fin del acuerdo sobre los textiles y el vestido? Revista de la Cepal, Santiago de Chile, Comisión Económica para América Latina y el Caribe (Cepal), n. 93 (LC/G.2347-P/E), dic. 2007.

HESTON, A.; SUMMERS, R.; ATEN, B. Penn World Table Version 6.2, Center for International Comparisons of Production, Income and Prices at the University of Pennsylvania, Sept. 2006.

HAUSMANN, R.; HWANG, J.; RODRIK, D. What you export matters. NBER Working Paper, n. 11905, Dec. 2005.

HARROD, R. International economics. Cambridge: Cambridge University Press, 1933.

JAYME JR., F.G. Balance-of-payments-constrained economic growth in Brazil. Revista de Economia Política, v. 23, jan./mar. 2003.

KALDOR, N. The case for regional policies, Scottish Journal of Political Economy, Nov. 1970.

KAPLAN, D. Finite mixture dynamic regression modeling of panel data with Implications for dynamic response analysis. Journal of Educational and Behavioral Statistics, v. 30, p. 169-187, 2005.

KRUGMAN, P. Desindustrialización, reindustrialización y tipo de cambio real. Estudios Económicos, El Colegio de México, Centro de Estudios Económicos, v. 3, n. 2, p. 149167, 1988.

LEÓN-LEDESMA, M.; THIRLWALL, A. P. The endogeneity of the natural rate of growth. Cambridge Journal of Economics, v. 26, p. 441-459, 2002.

LOPEZ, J.; CRUZ, A. Thirlwall's law and beyond: the Latin American experience. Journal of Post Keynesian Economics, v. 22, n. 3, Spring, 2000.

MCCOMBIE, J.; THIRLWALL A. P. Economic growth and the balance of payments constraint. New York: St. Martin's Press, 1994.

MIOZZO, M.; WALSH, V. International competitiveness and technological change. Oxford University Press, 2006. 
MORENO-BRID, J. C. Capital flows, interests payments and the balance-of-paymentsconstrained growth model: a theoretical and empirical analysis. Metroeconomica, v. 54, n. 2, 2003.

OCAMPO, J. A. The quest for dynamic efficiency: structural dynamics and economic growth in developing countries. In: OCAMPO, J. A. (Ed.). Beyond reforms: structural dynamics and macroeconomic theory. Stanford University Press, 2005.

PESARAN, M.; SMITH, R. Estimating long-run relationships from dynamic heterogeneous panels. Journal of Econometrics, Elsevier, v. 68, n. 1, p. 79-113, Jul. 1995.

PORCILE, G.; HOLLAND, M.; CIMOLI, M.; ROSAS, L. Especialización, tecnología y crecimiento en el modelo Ricardiano. Nova Economia, v. 16, n. 3, p. 485-506, 2006.

PREBISCH, R. Capitalismo periférico: crisis y transformación. Fondo de Cultura Económica, 1981.

PUGNO, M. A Kaldorian model of economic growth with labour shortage and major technical changes. Structural Change and Economic Dynamics, 7, p. 429-446, 1996.

RODRIGUEZ, O. El estructuralismo latinoamericano. México: Siglo XXI, 2007.

ROS, J. Development theory and the economics of growth. Michigan University Press, 2000.

SCHWARZ, G. Estimating the dimension of a model. Annals of Statistics, 6, 1978.

SETTERFIELD, M. Rapid growth and relative decline: modeling macroeconomic dynamics with hysteresis. London: McMillan, 1997.

THIRLWALL, A. The balance of payments constrain as an explanation of international growth rates differences. Banca Nazionale del Lavoro Quarterly Review, v. 128, 1979.

THIRLWALL, A. P. The nature of economic growth. Aldershot: Edward Elgar, 2002. 